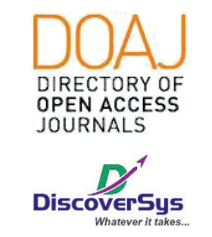

Published by DiscoverSys

\section{Neuroendocrine tumor of the gastrointestinal: report of two cases}

\author{
Made Agus Dwianthara Sueta, ${ }^{1 *}$ Made Oka Sastrawan ${ }^{2}$
}

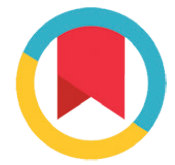

CrossMark

\section{ABSTRACT}

Introduction: Neuroendocrine tumors are malignancies that need special attention because they provide clinical manifestations that are not typical of excess gastrointestinal endocrine production. Case reports of neuroendocrine tumors are needed to be able to recognize the symptoms and determine the appropriate therapy.

Case series: The first patient was 49 years old Balinese female, consulted from internist with right lower-quadrant tenderness with fever for three days before. The patient also had diarrhea for one week before. The patient has done an appendectomy, and the pathological result of the appendix specimen was Neuroendocrine Appendix. The second patient was 62 years old Balinese male, complained about intermittent diarrhea for two months. Patient has done laparotomy exploration and colostomy because of a transverse colon tumor. The pathological result of the specimen from transverse colon tumor was mixed adeno-neuroendocrine carcinoma. Both patients have been treating with hormonal therapy (somatostatin analogs) long-acting release (LAR); the second patient also had combined with folfox IV chemotherapy.

Conclusion: The choice of therapy with somatostatin analogs in the first case and surgery in the second case gave a good response after treatment. However, in both cases, it does not guarantee the cure of this disease.
'Digestive Surgery Division, Surgery Department of Faculty of Medicine Udayana University, Sanglah General Hospital Denpasar, Bali-Indonesia

${ }^{2}$ Surgical Resident, Surgery Department of Faculty of Medicine Udayana University, Sanglah General Hospital Denpasar, BaliIndonesia
*Corresponding author: Made Agus Dwianthara Sueta, Digestive Surgery Division, Surgery Department of Sanglah General Hospital Denpasar, Bali-Indonesia, J. Diponegoro, Dauh Puri Klod, Denpasar Barat, Denpasar, Bali 80113

dwiantharasuetaagus@gmail.com

Received: 2019-02-03

Accepted: 2019-02-05

Published: 2019-08-01

Keywords: carcinoid, neuroendocrine tumor, somatostatin

Cite This Article: Sueta, M.A.D., Sastrawan, M.0. 2019. Neuroendocrine tumor of the gastrointestinal: report of two cases. Intisari Sains Medis 10(2): 359-362. DOI: 10.15562/ism.v10i2.409

\section{INTRODUCTION}

Gastrointestinal neuroendocrine tumors or carcinoid tumors contribute $2 \%$ of all digestive system malignancies. Most cases do not provide clear clinical manifestations even though the symptoms that appear in the case of neuroendocrine tumors can show specific symptoms based on hormones secreted. Diarrhea is one of the most common symptoms in neuroendocrine cases. ${ }^{1,2}$

\section{CASE 1}

A 49 years old Balinese female, was consulted from internist because of right lower-quadrant tenderness with fever for three days before. The patient also had nausea and vomiting. The pain was not released even she took a painkiller. The patient also had diarrhea for one week before. McBurney's point tenderness without rebound tenderness was noted. Further examination with ultrasonography, swollen appendix with wall thickening was found. Inflamed appendix with local peritonitis was found intraoperatively. Histopathological examination was carried out on appendix samples and showed a description of NET (neuroendocrine tumor) with infiltration of mesoappendix. The right hemicolectomy procedure is recommended for patients in the second operation, but she refused that. After surgery, the patient had hormonal therapy (somatostatin analogs) long-acting release (LAR) every four weeks for twelve times. After had the second hormonal therapy, the patient got no more diarrhea like before.

\section{CASE 2}

A 62 years old Balinese male, complained about severe abdominal pain with abdominal distention for two days before admission. He could not fart since one day before admission and also felt distention getting worse. Two months before, the patient got intermittent diarrhea but stopped itself with nothing to do for two days before admission, and the tenderness and distention started. On physical examination, we found abdominal distention and tenderness on the whole quadrant of the abdomen with stable hemodynamics. The bowel sound was increasing and sometimes heard the metallic sounds. We also found rectal ampullae was a collapse by rectal examination. Abdominal X-Ray demonstrated the colon was filled with gas and dilated to the level of the proximal colon. The patient was diagnosed with total ileus obstruction based on the colonoscopy examination. Laparotomy exploration was performed. Intraoperatively there was transverse colon tumor with dilatation at the proximal side. We decided to do a biopsy then made a colostomy. 


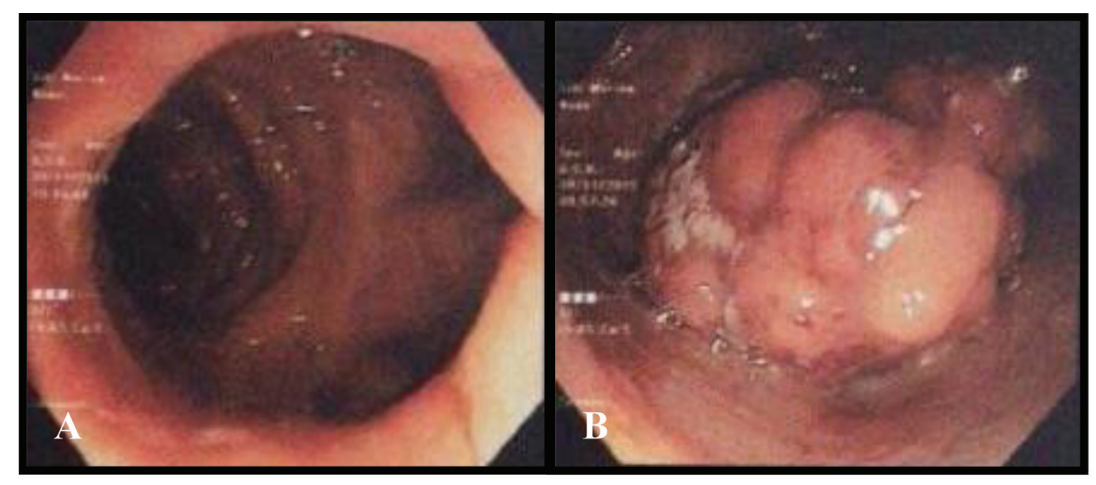

Figure 1 Colonoscopy examination showed ileus obstruction. A) Colon descending level. B) Total ileus obstruction on the transverse colon

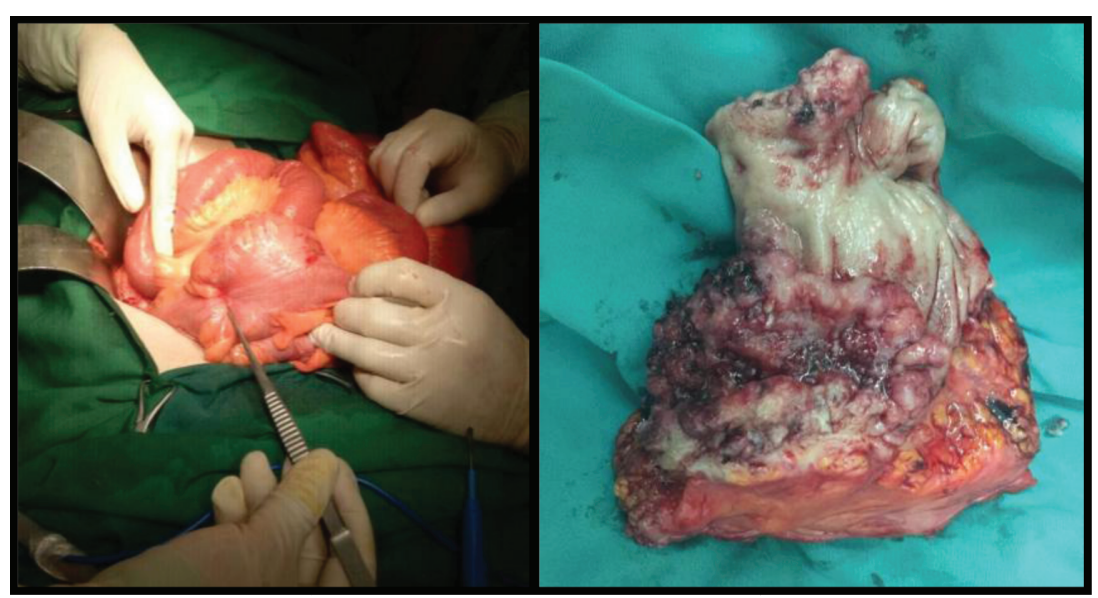

Figure 2 Laparotomy exploration. A) Transverse colon tumor with dilatation at proximal side. B) Resected transverse colon

The histopathological examination demonstrated a Neuroendocrine Carcinoma high grade (G3) infiltrated to mucosal (pT4). Immunohistochemistry examination showed a Mixed Adeno Neuroendocrine Carcinoma (MANEC). We gave combination therapy for this patient with hormonal therapy (somatostatin analogs) long-acting release (LAR), and Folfox IV Chemotherapy. After had two serial therapy, this patient was shown a good response.

\section{DISCUSSION}

Carcinoid term for gastrointestinal tract carcinoma-like lesions introduced by Oberndorfer in 1970, which showed more benign behavior than conventional malignancy. Thus, appendix carcinoid classified as (well-differentiated) neuroendocrine tumors (NET) in 2000 by WHO. This neoplasm more specific found in the lung and gastrointestinal tract. This tumor is specifically found in the digestive system and lungs because it comes from the neuroendocrine tissue. It was classified into foregut, midgut, and hindgut tumors. Foregut tumors include respiratory tract, stomach, thymus, pancreas, and duodenum. Midgut tumors include (appendix, right colon, and small intestine). Hindgut tumors include (transverse colon, descending colon, sigmoid, and rectum) . $^{3-5}$

Appendix carcinoid is the most common primary malignant lesion originating in the appendix. In the patients undergoing an appendectomy, $0.3-0.9 \%$ appendix carcinoid was found. Interestingly, female patients were recorded with a slightly higher incidence in an epidemiological study with appendix carcinoid; some authors ascribed to the higher appendectomy and laparoscopy rate. ${ }^{1,3}$ Detection more carcinoid lesion from these procedures could not fully explain higher carcinoid prevalence in female patients despite the higher surgery rate. Carcinoid appendix diagnosed with a peak incidence between $15-19$ years old for female and $20-29$ years old for male. However, appendix malignant lesions are concentrated in patients between $38-49$ years old. ${ }^{3,6}$

Carcinoid cell has the same progenitor with gastrointestinal cell but not in neural tissue. Even though the evidence suggested that carcinoid generally are malignant, carcinoid of the appendix have a better prognosis compared with another gastrointestinal carcinoid. Different cell derivation of the appendix compared to other gastrointestinal NET are the main reason despite both carcinoids still considered as malignancy. ${ }^{3-6}$ Carcinoid of appendix originated from the subepithelial neuroendocrine cell (SNC), whereas neuroendocrine epithelial cell (ENC) is the origin of another location carcinoid. Different $\beta$-catenin level, catestatin-A demonstrated in carcinoid of appendix compared to other gastrointestinal NET, ${ }^{7,9,10}$

Appendix carcinoid does not have a specific symptom or clinical sign. Most appendix carcinoid is asymptomatic and found incidentally during appendectomy, cholecystectomy or other benign pelvic surgery. Appendix carcinoid mimics more than 50\% acute appendicitis with the classical abdominal pain in the right lower quadrant. ${ }^{7-10}$ Tumor obstruction causes appendicitis symptom suggested by some author, but the only $25 \%$ of the obstructive factor was confirmed by the histopathological analysis. Even asymptomatic obstructive carcinoid of appendix identified during CT-scan or ultrasound examination as appendicitis, and there is only one reported case presenting NET of the appendix from sonographic diagnosis in a 14-year-old boy. However, these techniques routine application is very doubtfully will increase the preoperative diagnosis. In rare carcinoid syndrome, hematological, biochemical and urinary examination are the only indicated to monitor the disease progression. ${ }^{8-10}$

NEC (neuroendocrine carcinoma) occurrence in the colorectal is rare and has been reported from 
$0.3 \%$ to $3.9 \%$ of all colorectal cancer, and comprises from $10 \%$ to $34 \%$ of colon undifferentiated carcinoma. Different terms used to describe this type of tumors such as pleomorphic, atypical, or malignant carcinoid, small cell undifferentiated carcinoma, small cell neuroendocrine carcinoma, or stem cell carcinoma..$^{3-6}$ Gastrointestinal tract neuroendocrine tumors comprise a pathological spectrum ranging from carcinoid to extremely aggressive neuroendocrine carcinoma. ${ }^{1,2}$ NEC classified into three subtypes, well differentiated, intermediate, and small cell-type regarding cell type. In this study, our cases thus classified as an intermediate type according to the guideline. However, none of these studies demonstrated clinical and pathological differences between those subtypes.

On the other hand, the small cell type of NEC showed a poorer prognosis compared to the other two types. Moreover, according to the histologic pattern they classified NEC as pure neuroendocrine with the poorer survival rate, predominant neuroendocrine, and equal neuroendocrine and exocrine. In this study, our cases considered as a pure neuroendocrine subtype and predicted to have a poor survival rate. ${ }^{13}$

Attempting curative resection is considered as the initial approach to the gastrointestinal neuroendocrine tumors. Omeprazole and octreotide as the new medical treatments demonstrated better control for endocrine syndromes for undergoing elective surgery patients. However, symptomatic carcinoid tumor majority will have metastases and won't be curable. Reoperation to debulk symptomatic metastatic disease often justified since this tumor usually grows in slow progression. However, life-saving surgery does not cure this disease. Therefore medical palliation is often necessary. Standard supportive therapy can be used to treat mild symptoms such as pain or diarrhea. However, more aggressive and specific therapy become necessary to eliminate the tumor and its hormonal product. ${ }^{2,3}$

\section{Regional therapy and surgery}

Debulking surgery to reduce the bulk of a tumor is the most definitive option for isolated hepatic metastases case. A Mayo Clinic series demonstrated $73 \%$ survival and $30 \%$ symptom-free survival at four years from the 70 patients with NET who underwent hepatic resection. Morbidity rate was minimal from the intervention in the highly selected group. A matched control patients retrospective review demonstrated a significant result in 5 -years survival ( $73 \%$ vs. $29 \%$ ) from hepatic resection patients. The relationship between patient's prognosis and disease burden was the main result demonstrated in this study despite the selection bias that flawed this study. ${ }^{1,4}$

\section{Hormonal Therapy}

Somatostatin analogs become a medical revolution in handling neuroendocrine tumor cases since the late 1970s. Native somatostatin binds to receptors on the cell surface membrane, which are present on gastrointestinal and endocrine tissue in an extensive number and their malignant counterparts. ${ }^{11,12}$ Activation of the receptor dramatically inhibits neuroendocrine vesicle activity and decrease the secretory production in the pancreas, stomach, and intestines. However, somatostatin half-life is only one minute, making it ineffective for clinical use. Lanreotide and octreotide (long-acting synthetic octapeptides) is a therapeutic choice that is used because these analogs are clinically practical. Dramatic benefits have been demonstrated in multiple clinical trials in treating PETs and carcinoid syndromes. Reduction of 70\% flushing episodes and $50 \%$ diarrhea on carcinoid syndrome with either analog reported in most studies. Chromogranin A serum and urinary 5-HIAA level measurement as biochemical responses occur in the order of $40 \%$ to $70 \%$. Somatostatin analogs administration reported having the ability in anecdotal tumor regressions. ${ }^{1,3}$

However, objective partial responses in neuroendocrine tumors not reported in a prospective series of 103 patients from the German Sandostatin Multicentre Study Group. Interestingly, Interestingly, up to one-third of patients experienced stabilization of the disease for at least three months with $10 \%$ having disease stabilization for three years. A second study from Memorial Sloan Kattering involving 34 patients demonstrated disease stabilization treated with octreotide up to $50 \%$ of the patients. These agents are impractical to administer as they require subcutaneous injection up to three times a day despite extremely useful in treating neuroendocrine tumor symptoms have been proved by the somatostatin analogs. ${ }^{3}$

Report of the Long-acting release lanreotide usage in 39 patients with carcinoid syndrome showed symptomatic and biochemical response from trials using daily subcutaneous injection. A $30 \mathrm{mg}$ intramuscular drug administered every two weeks and only induced transient discomfort on the injection site. ${ }^{14}$

\section{Chemotherapy}

The treatment of gastrointestinal tumor and their syndromes using chemotherapy promising mixed success. A unique agent, streptozocin, show ability effect specifically on the neuroendocrine pancreatic islet cells. This agent has been combined with 
doxorubicin, epirubicin, dacarbazine, and fluorouracil. These combinations can reduce the tumor progression in more than $60 \%$ patients with PETs, but less response in carcinoid tumor, also the toxicity is significant enough to preclude their use as palliative agents. The randomized clinical trial seems impractical because of this tumor rarity rate, and it is still unclear chemotherapy agents combination offer a survival benefit for the treated patients. ${ }^{1,2,15}$

\section{CONCLUSIONS}

Recognizing symptoms and early biopsy checks will improve outcomes even though the case of neuroendocrine tumors is a rare case. Somatostatin analogs and surgery provide a good response in both cases but have not been able to confirm the cure of the disease. Further research on this disease and specific antitumor therapy still needs to be done.

\section{CONFLICT OF INTEREST}

None declared.

\section{FUNDING}

Own funding.

\section{REFERENCES}

1. Toole DO., Kianmanesh R., Caplin M. Consensus Guidelines for the Management of Patients with Digestive Neuroendocrine Tumors: An Update. Neuroendocrinology. 2016;103:117-118.

2. Castaño JP., Sundin A., Maecke HR., Villabona C., VazquezAlbertino R., Navarro E., Oberg K. Gastrointestinal neuroendocrine tumors (NETs): new diagnostic and therapeutic challenge. Cancer Metastasis Rev. 2014;33(1):353-9.

3. Narayanan, S., Kunz PL. Role of Somatostatin Analogues in the Treatment of Neuroendocrine Tumors. Hematol Oncol Clin N Am. 2016;30:163-177.
4. Griniatsos J., Michail O. 2014. Appendiceal neuroendocrine tumors: Recent insight and clinical implications. World Journal of Gastrointestinal Oncology. 2014;2:192-196.

5. Moosavy, SH., Andrabi Y., Esmaeeli S., Amini A. Small bowel obstruction by a terminal ileum carcinoid tumor: a case report. Medical Journal of Islamic Republic of Iran. 2011;25(3):165-169.

6. Xenaki S., Lasithiotakis K., Andreou A., Aggelaki S., Tzardi M., Daskalaki A., Chalkiadakis G., Chrysos E. Case report: A Rare Case of Mixed Neuroendocrine Tumor and Adenocarcinoma of the Pancreas. Case Reports in Surgery. 2016;2016:1-4.

7. Prasad ND., Manmadharao V., Babji K., Reddy DK. Intestinal Obstruction by Carcinoid Tumor in Ileum: A Case Report. International Journal of Scientific Study. 2015;2(10):166-168.

8. Uccella S., Sessa F., La Rosa S. Diagnostic Approach to Neuroendocrine Neoplasm of Gastrointestinal Tract and Pancreas. Turk Patoloji Derg 2015;31(Suppl):113-127.

9. Padhi S., Sahoo B., Meher P., Besra K., Samantaray S. Neuroendocrine Tumors - A Case Series and Literature Review. International Journal of Medical Research and Pharmaceutical Sciences. 2017;4(2):11-17.

10. Kim JY., Hong SM. Recent Updates on Neuroendocrine Tumors From the Gastrointestinal and Pancreatobiliary Tracts. Arch Pathol Lab Med. 2016;140:437-448.

11. Kumar U., Michael G. Somatostatin and Somatostatin Receptors. Result and problems in cell differentiation. 2009;50:137-84.

12. Wolin EM. The Expanding Role of Somatostatin Analogs in the Management of Neuroendocrine Tumors. Gastrointest Cancer Res.2102;5:161-168.

13. Saclarides TJ., Szeluga D., Staren ED. Neuroendocrine cancers of the colon and rectum. Results of a ten-year experience. Dis Colon Rectum. 1994;37(7):635-42

14. Ruszniewski P., Ish-Shalom S., Wymenga M., O’Toole D., Arnold R., Tomassetti P., Bax N., Caplin M., Eriksson B., Glaser B., et al. Rapid and sustained relief from the symptoms of carcinoid syndrome: results from an open 6-month study of the 28-day prolonged-release formulation of lanreotide. Neuroendocrinology. 2004;80:244-251.

15. M Indrayani LPI., Sriwidnyani NP. K-RAS mutation profile in colorectal carcinoma patients in Sanglah Hospital Denpasar, Bali-Indonesia. Bali Med J. 2017;3(3):S40-S42 doi: 10.15562/bmj.v3i3.717.

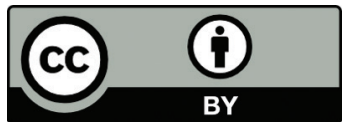

This work is licensed under a Creative Commons Attribution 